\title{
Nuclear $\gamma$ resonance time-domain interferometry: Quantum beat and radiative coupling regimes compared in revealing quasielastic scattering
}

\author{
G. V. Smirnov, ${ }^{1, *}$ U. van Bürck, ${ }^{2}$ H. Franz, ${ }^{3}$ T. Asthalter, ${ }^{4}$ O. Leupold, ${ }^{5}$ E. Schreier, ${ }^{5}$ and W. Petry ${ }^{2}$ \\ ${ }^{1}$ Russian Research Center "Kurchatov Institute," 123182 Moscow, Russia \\ ${ }^{2}$ Physik-Department, Technische Universität München, D-85748 Garching, Germany \\ ${ }^{3}$ Hamburger Synchrotronstrahlungslabor, Deutsches Elektronen Synchrotron, D-22603 Hamburg, Germany \\ ${ }^{4}$ Institut für Physikalische Chemie, Universität Stuttgart, D-70569 Stuttgart, Germany \\ ${ }^{5}$ European Synchrotron Radiation Facility, F-38043, Grenoble, France
}

(Received 13 February 2006; published 25 May 2006)

\begin{abstract}
Nuclear $\gamma$ resonance time-domain interferometry (TDI) is a method where the interference pattern is built in time during the delayed scattering of synchrotron radiation by a system composed of two nuclear resonant targets and a nonresonant sample placed in between. The radiation transmitted through the upstream target is scattered by the sample at a finite angle and is transmitted through the downstream target. Atomic motions in the sample perturbing the delayed radiation can be revealed directly in the time interference pattern. The unique sharpness of nuclear resonant scattering allows one to investigate atomic motions proceeding for times in the range of nanoseconds to microseconds. The radiative coupling (RC) regime of TDI where the radiation from the upstream nuclear target is in resonance with the downstream target was investigated experimentally and compared with the quantum beat $(\mathrm{QB})$ regime where the resonances in the targets are well separated. Clear evolutions of the interference patterns were observed with glycerol as a sample in both regimes, manifesting the increase of quasielastic scattering both with increasing temperature and with momentum transfer. However, the increase of quasielastic scattering is revealed in quite different ways: in the QB regime through pronounced changes of the quantum beat modulation of a fixed interference pattern, in the RC regime via strong changes of the interference pattern itself, mainly of its dynamical beat structure. It was possible to find relaxation parameters by which the two sets of completely different time evolutions for the QB and RC regimes were consistently fitted. Such a treatment will in the future considerably enlarge the dynamic range of the method and increase the reliability of the data analysis.
\end{abstract}

DOI: 10.1103/PhysRevB.73.184126

PACS number(s): $61.10 . \mathrm{Eq}, 76.80 .+\mathrm{y}, 42.25 . \mathrm{Hz}$

\section{NUCLEAR $\gamma$ RESONANCE TIME-DOMAIN INTERFEROMETRY}

Quantum interference is a typical property of nuclear resonance scattering of Mössbauer and synchrotron radiation (SR) by nuclei in solid matter (e.g., see Ref. 2). The interference takes place in both the real-space and time domains. In real space the scattering paths are related to coherent scattering by different nuclei. For instance, spatial multipath interference results in the coherent, forward-directed response of a sample containing ensembles of nuclei. In the time domain the interference of different spectral components of the radiation specifies the temporal response of the sample. Here the different scattering paths are determined by the distribution of the resonance energy in the sample-i.e., by the resonance shape and by the splitting of the nuclear level into hyperfine components. The coherent nuclear scattering of a SR pulse in the time domain has been described analytically for Bragg reflection ${ }^{3-5}$ and forward scattering. ${ }^{5}$ The results obtained in theory refer to the response of a single resonant target.

For the solution of some physical problems, composite scattering systems are required, most frequently these are systems of two resonant targets, situated upstream and downstream in the beam. For example, a two-target system was used to demonstrate quantum beats, ${ }^{6}$ or to determine the chemical shift between the targets, ${ }^{7}$ or to reveal and to study the perturbation in the scattering system caused by ultra- sound applied to one of the targets. ${ }^{8-11}$ Two-target systems were also used in Mössbauer heterodyne spectroscopy ${ }^{12}$ and phase determination ${ }^{13,14}$ to obtain energy spectra of the transmitted radiation. To describe the scattering from a composite system it is natural to present its response in the form of a coherent superposition of a few scattering paths related to the scattering of radiation by the constituent targets. ${ }^{2,15}$

In fact a system of two targets works as a time-domain interferometer where the targets can be considered as the interferometer arms. With the help of one of the targets the emission from the other target can be probed both in phase and in amplitude in time (for illustration see, e.g., Fig. 19 in Ref. 2). A fruitful development of the concept of a twonuclear-target system was to extend this idea to also studying dynamics in nonresonant samples. This was realized by placing a nonresonant sample in the scattering geometry between the nuclear targets. Quasielastic scattering from the sample can be investigated this way. ${ }^{1,16,17}$ With the help of this technique the dynamics of the electron density in a medium can be revealed directly in the time domain. ${ }^{1,15,18,19}$

Two regimes of the nuclear interferometer can be clearly distinguished: those in the absence and in the presence of the radiative coupling between interferometer arms. Radiative coupling (RC) occurs when the radiation from nuclei in the upstream target is in resonance with the nuclei in the downstream target. The strongest RC takes place at zero separation of the resonances in the two nuclear targets. This regime is called the $R C$ regime of the interferometer. In the case of a 


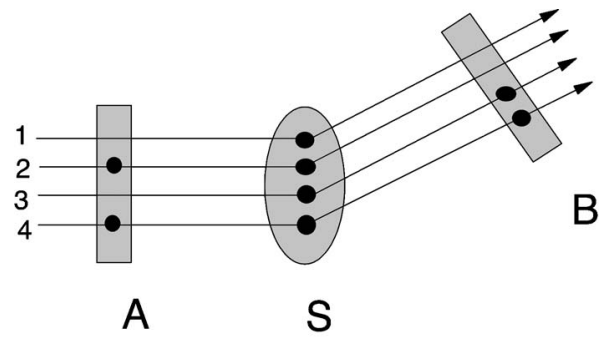

FIG. 1. Scattering paths of the radiation through resonant targets $A$ and $B$ and nonresonant sample $S$ in the interferometer.

large separation of the resonances, the radiative coupling vanishes and one comes to a pure quantum beat solution. Here the oscillatory behavior of the intensity occurs at the frequency determined by the separation of the resonances. This regime is called the $Q B$ regime of the interferometer.

In the present paper the two regimes of time-domain interferometry (TDI) were compared in experiment. As a model sample, viscous glycerol was used which exhibits structural relaxation on the considered time scale near the transition towards its glassy state. The relaxation gives rise to quasielastic scattering of the radiation in the sample. The scattering related to slow atomic motion-i.e., on the time scale of nuclear resonant scattering - can be directly revealed with the help of TDI. For the given time structure of thirdgeneration SR sources, which is composed of 100-ps pulses separated by $100-700 \mathrm{~ns}$, the Mössbauer isotope ${ }^{57} \mathrm{Fe}$ is ideally suited to reveal relaxation with characteristic times in the range of nanoseconds to microseconds.

In Sec. II a brief overview of the main results of the theory ${ }^{15}$ is given. Section III is devoted to a description of the experimental details, to an analysis of the experimental results, and to the conclusions.

\section{QUANTUM BEAT AND RADIATIVE COUPLING REGIMES OF NUCLEAR RESONANCE INTERFEROMETRY}

In Fig. 1 a typical scattering geometry met in the nuclear resonant interferometer is displayed. The radiation transmitted through target $A$ is scattered by sample $S$ and finally transmitted through target $B$.

Resonant targets $A$ and $B$ are the interferometer arms, which are supposed to be identical and to have a single line structure of the resonance. We call the response of a nuclear target $G(t)$ and that of the sample $g(\mathbf{q}, t)$, where $\mathbf{q}$ is the momentum transfer for the scattering from the sample. The scattering in a nuclear target is delayed because of the large lifetime of the intermediate excited nuclear state. On the contrary, the scattering of radiation by electrons in a nonresonant sample occurs promptly, reflecting the instant state of the electron density distribution. Nevertheless, the response of the nonresonant sample is a time-dependent function because the electron density distribution inside the sample is varying due to atomic dynamics while the radiation from the upstream nuclear target is being scattered. In the case of forward scattering the delayed part of the function $G$ is given by $^{5}$

$$
G(t) \propto-\frac{T}{2 t_{0}} e^{-i \omega_{r} t-t / 2 t_{0}} \frac{J_{1}\left(\sqrt{T t / t_{0}}\right)}{\sqrt{T t / t_{0}}},
$$

where $T=n \sigma_{0} f$ is the effective resonance thickness of the target, with $n$ being the number of resonant nuclei per unit area, $\sigma_{0}$ the resonance cross section of the nuclear transition, and $f$ the Lamb-Mössbauer factor; $\omega_{r}$ is the resonance frequency, $t_{0}=\hbar / \Gamma_{0}$ is the lifetime of the nuclear excited state with natural energy level width $\Gamma_{0}$, and $J_{1}$ is the Bessel function of first kind and first order. The electronic absorption of the radiation in the nuclear targets is neglected for the sake of simplicity. It results only in a constant attenuation of the intensity.

The response of the nonresonant sample $g(\mathbf{q}, t)$ will be considered in the limit of weak scattering where the kinematical approximation of the scattering theory can be applied. In this case the sample response is proportional to the Fourier coefficient of the electron density: $g(\mathbf{q}, t) \propto R(\mathbf{q}, t)$ (see Ref. 15). The latter can be presented in solid matter as follows:

$$
R(\mathbf{q}, t)=\sum_{a} f_{a}(\mathbf{q}) e^{-i \mathbf{q} \cdot \mathbf{r}_{a}(t)}
$$

where $\mathbf{r}_{a}(t)$ is the time-dependent coordinate of the $a$ th atom and $f(\mathbf{q})$ is the atomic form factor. The function $R(\mathbf{q}, t)$ is actually the sum of the scattering amplitudes from the collection of atoms at a particular momentum transfer $\mathbf{q}$ and at the time $t$.

For our system the entire scattering process can be presented as the coherent superposition of the paths shown in Fig. 1, where the dark circles indicate the interaction of the radiation with a scatterer. Altogether four paths can be distinguished: (1) prompt scattering of the SR pulse by the sample without interaction with the nuclear targets, (2) delayed scattering of the SR pulse by the upstream nuclear target followed by prompt scattering by the sample, (3) prompt scattering of the SR pulse by the sample followed by delayed scattering by the downstream nuclear target, and (4) delayed scattering by the downstream target of the radiation scattered at first by the upstream target and the sample. Let the considered scattering paths be described by the probability waves $W_{1-4}(t)$, respectively.

The development of the scattering process in the interferometer is characterized by specific times, which are, e.g., for wave $W_{4}(t), t_{s}$ the start time set by the arrival of the SR pulse at the detector, at the same time the two nuclear targets are excited by the prompt radiation (the flight time of radiation in the free space of the system can be neglected), $\left(t_{s}+t^{\prime}\right)$ the time of emission from the upstream target with $0 \leq t^{\prime},\left(t_{s}\right.$ $+t^{\prime}$ ) also the time of scattering from the sample, as well as the time of excitation of the downstream target, and $\left(t_{s}+t\right)$ the time of emission from the downstream target with $t^{\prime} \leq t$.

The probability waves describing the scattering over the constituent paths are as follows (the SR pulse is presented by a $\delta$ function in time):

$$
\begin{gathered}
W_{1}(t)=g\left(\mathbf{q}, t_{s}\right) \delta(t), \\
W_{2}(t)=g\left(\mathbf{q}, t_{s}+t\right) e^{-i \Omega t} G(t),
\end{gathered}
$$




$$
\begin{gathered}
W_{3}(t)=G(t) g\left(\mathbf{q}, t_{s}\right), \\
W_{4}(t)=\int_{0}^{t} d t^{\prime} G\left(t-t^{\prime}\right) g\left(\mathbf{q}, t_{s}+t^{\prime}\right) G\left(t^{\prime}\right) e^{-i \Omega t^{\prime}},
\end{gathered}
$$

where the additional temporal phase $\Omega t$ accounts for the possible frequency shift between the resonances in the interferometer arms. Since the response of the nuclear target does not depend on the arrival time $t_{s}$, this time is omitted from the argument of the $G$ function. The whole scattering process is described by the probability wave

$$
W(t)=W_{1}(t)+W_{2}(t)+W_{3}(t)+W_{4}(t) .
$$

Equation (4) describes the interference of the considered scattering paths. If $\hbar \Omega$ exceeds essentially the resonance width $\Gamma_{0}$, the last wave is vanishing. Thus, while in the RC regime the interference of four scattering paths forms the scattering picture, in the QB regime only three paths should be taken into account to yield the final result.

In the case of a stiff sample, $g(\mathbf{q}, t)$ is a time-independent function, so that the time dependence of the probability wave $W(t)$ is determined only by the nuclear resonant scattering. However, in the presence of atomic motion in the sample the response function of the sample introduces an additional time dependence into the structure of the waves $W_{2}$ and $W_{4}$. In this way the resultant interference picture is affected by the atomic dynamics in the sample. The scattering intensity related to a single SR pulse is found as $I(t) \propto W(t) W^{*}(t)$. In the following expression for the intensity we leave only the delayed part:

$$
\begin{aligned}
I(t)= & |G(t)|^{2}\left\{\left|g\left(\mathbf{q}, t_{s}\right)\right|^{2}+\left|g\left(\mathbf{q}, t_{s}+t\right)\right|^{2}+2 \operatorname{Re}\left[g \left(\mathbf{q}, t_{s}\right.\right.\right. \\
& \left.\left.+t) g^{*}\left(\mathbf{q}, t_{s}\right) e^{-i \Omega t}\right]\right\}+2 \operatorname{Re}\left\{G ^ { * } ( t ) \left[g^{*}\left(\mathbf{q}, t_{s}\right)+g^{*}\left(\mathbf{q}, t_{s}\right.\right.\right. \\
& \left.\left.+t) e^{i \Omega t}\right] \int_{0}^{t} d t^{\prime} G\left(t-t^{\prime}\right) g\left(\mathbf{q}, t_{s}+t^{\prime}\right) G\left(t^{\prime}\right) e^{-i \Omega t^{\prime}}\right\} \\
& +\left|\int_{0}^{t} d t^{\prime} G\left(t-t^{\prime}\right) g\left(\mathbf{q}, t_{s}+t^{\prime}\right) G\left(t^{\prime}\right) e^{-i \Omega t^{\prime}}\right|^{2} .
\end{aligned}
$$

The responses $g\left(\mathbf{q}, t_{s}\right)$ and $g\left(\mathbf{q}, t_{s}+t\right)$ correspond to the microstates of the electron density in the sample encountered by a definite SR pulse at its passage through the system. To get the intensity measured in the experiment one has to average expression (5) over a large number of SR passages, because the atomic dynamics in the sample is not correlated with the arrival of an individual SR pulse. While averaging in the final expression for the scattering intensity appears the characteristic correlation function

$$
S(\mathbf{q}, t)=\frac{1}{t_{m}} \int d t_{s} \sum_{a} \sum_{a^{\prime}} f_{a}(\mathbf{q}) f_{a^{\prime}}(\mathbf{q}) e^{-i \mathbf{q} \cdot\left[\mathbf{r}_{a}\left(t_{s}+t\right)-\mathbf{r}_{a^{\prime}}\left(t_{s}\right)\right]}
$$

where $t_{m}$ is the measurement time covering the complete number of passed SR pulses. Under conditions of thermodynamic equilibrium the results of the averaging of the $\left|g\left(\mathbf{q}, t_{s}\right)\right|^{2}$ and $\left|g\left(\mathbf{q}, t+t_{s}\right)\right|^{2}$ terms in Eq. (5), which refer to particular times $t_{s}$ or $t_{s}+t$, are identical. They are determined only by the geometrical arrangement of the mean positions of the atoms. This result presents the structure factor of the sample. Thus, one has to deal only with the correlator containing different times, as shown in Eq. (6). The result of the calculation is known as the intermediate scattering function which is the time Fourier transform of the dynamic structure factor $S(\mathbf{q}, \omega)$. For real correlation functions $S(\mathbf{q}, t)$ the observed intensity can be written as follows:

$$
\begin{aligned}
\bar{I}(t)= & 2|G(t)|^{2}[S(\mathbf{q}, 0)+S(\mathbf{q}, t) \cos (\Omega t)]+4 G^{*}(t) \int_{0}^{t} d t^{\prime} G(t \\
& \left.-t^{\prime}\right) S\left(\mathbf{q}, t^{\prime}\right) G\left(t^{\prime}\right) \cos \Omega t^{\prime}+2 \int_{0}^{t} d t^{\prime} \int_{0}^{t^{\prime}} d t^{\prime \prime} G(t \\
& \left.-t^{\prime}\right) G\left(t^{\prime}\right) S\left(\mathbf{q}, t^{\prime}-t^{\prime \prime}\right) G^{*}\left(t-t^{\prime \prime}\right) G^{*}\left(t^{\prime \prime}\right) \cos \left[\Omega\left(t^{\prime}-t^{\prime \prime}\right)\right] .
\end{aligned}
$$

This expression is valid for an arbitrary shift of the nuclear resonances in the interferometer arms. The cases of interest refer to $\Omega \gg \frac{\Gamma_{0}}{\hbar}$ (QB regime) and $\Omega=0$ (RC regime). For the QB regime all integral terms disappear due to the fast oscillations of the integrands so that one arrives at the expression

$$
\bar{I}(t)=2|G(t)|^{2}[S(\mathbf{q}, 0)+S(\mathbf{q}, t) \cos (\Omega t)] .
$$

In the absence of any atomic dynamics in the sample the intensity is given by $2 S(\mathbf{q}, 0)|G(t)|^{2}[1+\cos (\Omega t)]$. So in the case of a stiff sample it is the doubled intensity of a single nuclear target which is reduced by the finite reflectivity from the sample and is modulated in time by the cosine function at frequency $\Omega$. In the presence of atomic dynamics the modulation amplitude is modified by the time-dependent intermediate scattering function $S(\mathbf{q}, t)$. Just the time-dependent modulation of the oscillation amplitude contains information about the atomic dynamics in the sample.

In the RC regime all cosines in Eq. (7) are replaced by unity and one obtains the following expression:

$$
\begin{aligned}
\bar{I}(t)= & 2|G(t)|^{2}[S(\mathbf{q}, 0)+S(\mathbf{q}, t)]+4 G^{*}(t) \int_{0}^{t} d t^{\prime} G(t \\
& \left.-t^{\prime}\right) S\left(\mathbf{q}, t^{\prime}\right) G\left(t^{\prime}\right)+2 \int_{0}^{t} d t^{\prime} \int_{0}^{t^{\prime}} d t^{\prime \prime} G\left(t-t^{\prime}\right) G\left(t^{\prime}\right) S\left(\mathbf{q}, t^{\prime}\right. \\
& \left.-t^{\prime \prime}\right) G^{*}\left(t-t^{\prime \prime}\right) G^{*}\left(t^{\prime \prime}\right)
\end{aligned}
$$

In the case of a stiff sample the scattering intensity corresponds now to the coherent response of the nuclear target having the doubled thickness $2 T$. The intensity is reduced by the reflectivity of the static sample, $S(\mathbf{q}, 0)$. The atomic dynamics in the sample manifests itself now in a more complicated way: the intermediate scattering function $S(\mathbf{q}, t)$ enters each term of Eq. (9). In particular, the atomic motion brings a perturbation into the radiative coupling between the interferometer arms and perturbs the direct nuclear scattering from the upstream nuclear target. As a consequence, the overall shape of the time dependence of the scattering is modified.

Obviously, the effect of the perturbation is determined by the specific form of the intermediate scattering function. In 


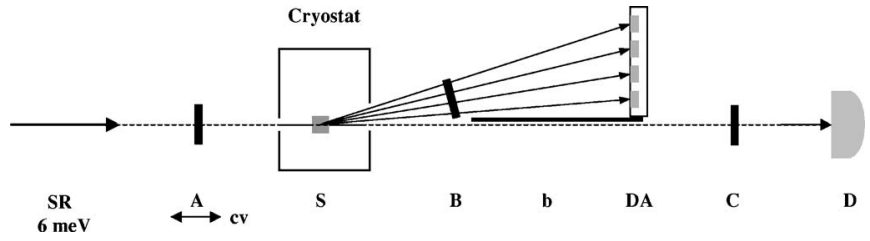

FIG. 2. Scheme of the experimental setup. The interferometer consists of the stainless-steel targets $A$ and $B$, the nonresonant glycerol sample $S$ in the cryostat, and the detector array $D A$ shielded by a baffle $b$. The motion of target $A$ on the constantvelocity drive is monitored by nuclear forward scattering through targets $A$ and $C$ measured by detector $D$.

turn, the latter is directly related to the character of the atomic motion. Usually a motion can be decomposed into a fast motion of the atoms around their mean positions (thermal vibrations with characteristic period $10^{-13} \mathrm{~s}$ ) and a slow motion of the mean positions in the sample due to diffusion, structural relaxation, etc. The motion is regarded as slow in this context, when it develops in the time scale of the nuclear scattering process (in the case of nuclear resonance in ${ }^{57} \mathrm{Fe}$ $\approx 10^{-7} \mathrm{~s}$ ). The average velocity of the slow motion leads to a small displacement of the atomic mean position (of order of the wavelength of the radiation) during the observation time. The great difference in the characteristic times of the fast motion and of the process of nuclear scattering does not allow one to observe the development of the fast motion by means of TDI. Its only effect is the reduction of the total scattering intensity by the Debye-Waller factor $f_{D W}$, which is obtained while averaging over the fast thermal oscillations of the atoms. As to the slow motion it can definitely be revealed with respect to its development in space and time with the help of TDI. This motion results in a $q$-dependent decay of the intermediate scattering function with time just during the nuclear scattering process. By means of the $q$ dependence its spatial development can be revealed.

The behavior of the intermediate scattering function is strongly determined by the specific type of atomic motion under study. In the double sum of Eq. (6) it is convenient to discuss separately the terms with $a=a^{\prime}$ and those with $a$ $\neq a^{\prime}$. The first sum $\Sigma_{a} e^{-i \mathbf{q} \cdot\left[\mathbf{r}_{a}\left(t_{s}+t\right)-\mathbf{r}_{a}\left(t_{s}\right)\right]}$ presents the correlation of an atom with itself in space at different times, averaged over all atoms. The second sum $\Sigma_{a} \sum_{a^{\prime}} e^{-i \mathbf{q} \cdot\left[\mathbf{r}_{a}\left(t_{s}+t\right)-\mathbf{r}_{a^{\prime}}\left(t_{s}\right)\right]}$ presents the correlation of an atom with another one in space and in time, averaged over all pairs of atoms. These are the self-part and the distinct part of the pair correlation function introduced and analyzed in general form by van Hove. ${ }^{20}$ If the motion of different atoms is uncorrelated, we obtain for nonzero $\mathbf{q}$ that all terms in the last double sum are randomly phased and thus cancel each other in the average. Consequently, in the case of random motion of atoms, we may neglect the term describing the interatomic space correlations. In this case only the van Hove self-correlation function is left to modify the time interference pattern. Otherwise the pair correlation function reveals itself.

\section{$140 \mathrm{~K}$}
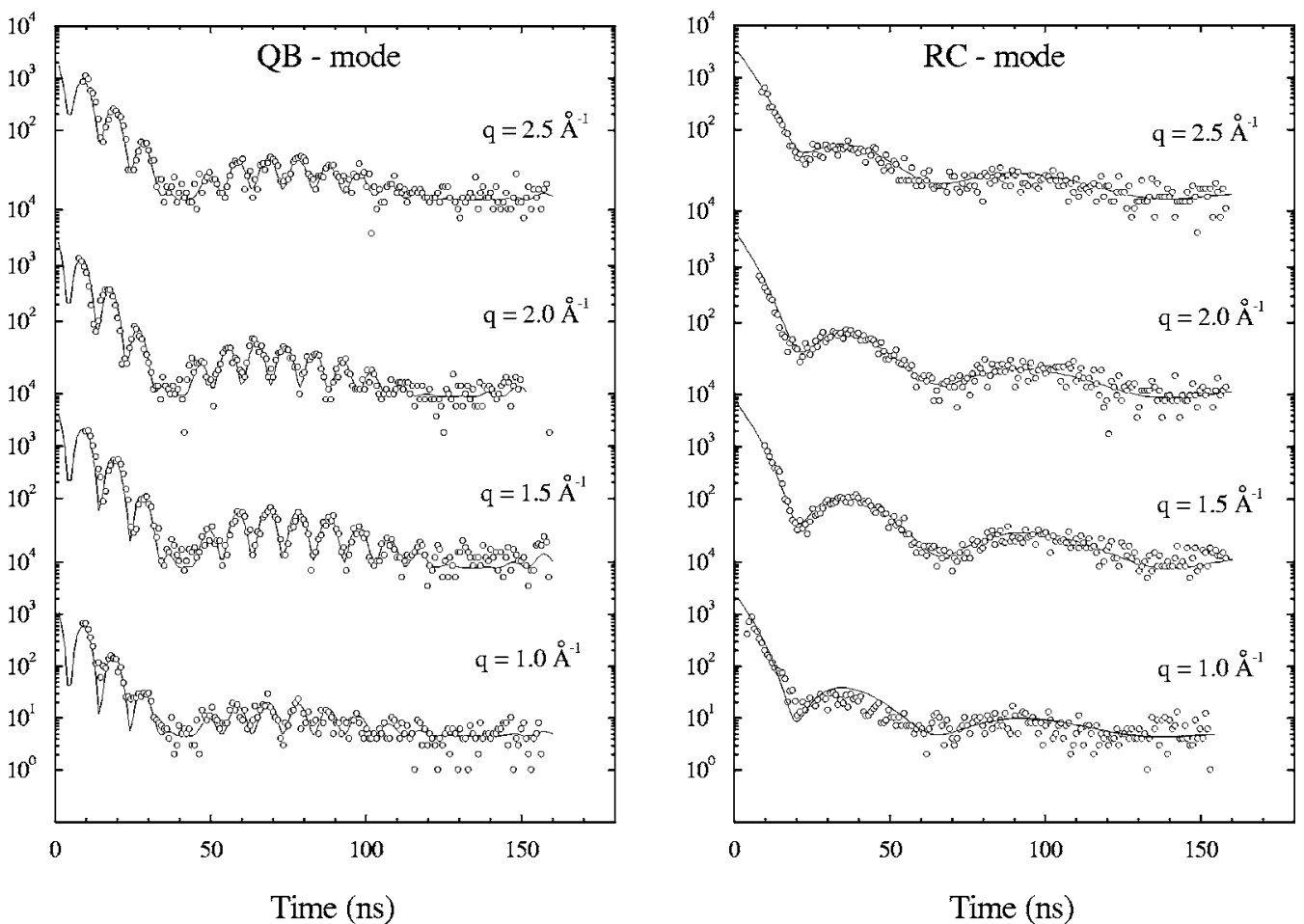

FIG. 3. Time dependences of scattering of the synchrotron radiation in the interferometer measured in the QB (left-hand panel) and RC (right-hand panel) regimes at the temperature of the glycerol sample $140 \mathrm{~K}$ and at different momentum transfers. The solid curves are simulations obtained on the basis of Eqs. (8) and (9). 
$255 \mathrm{~K}$
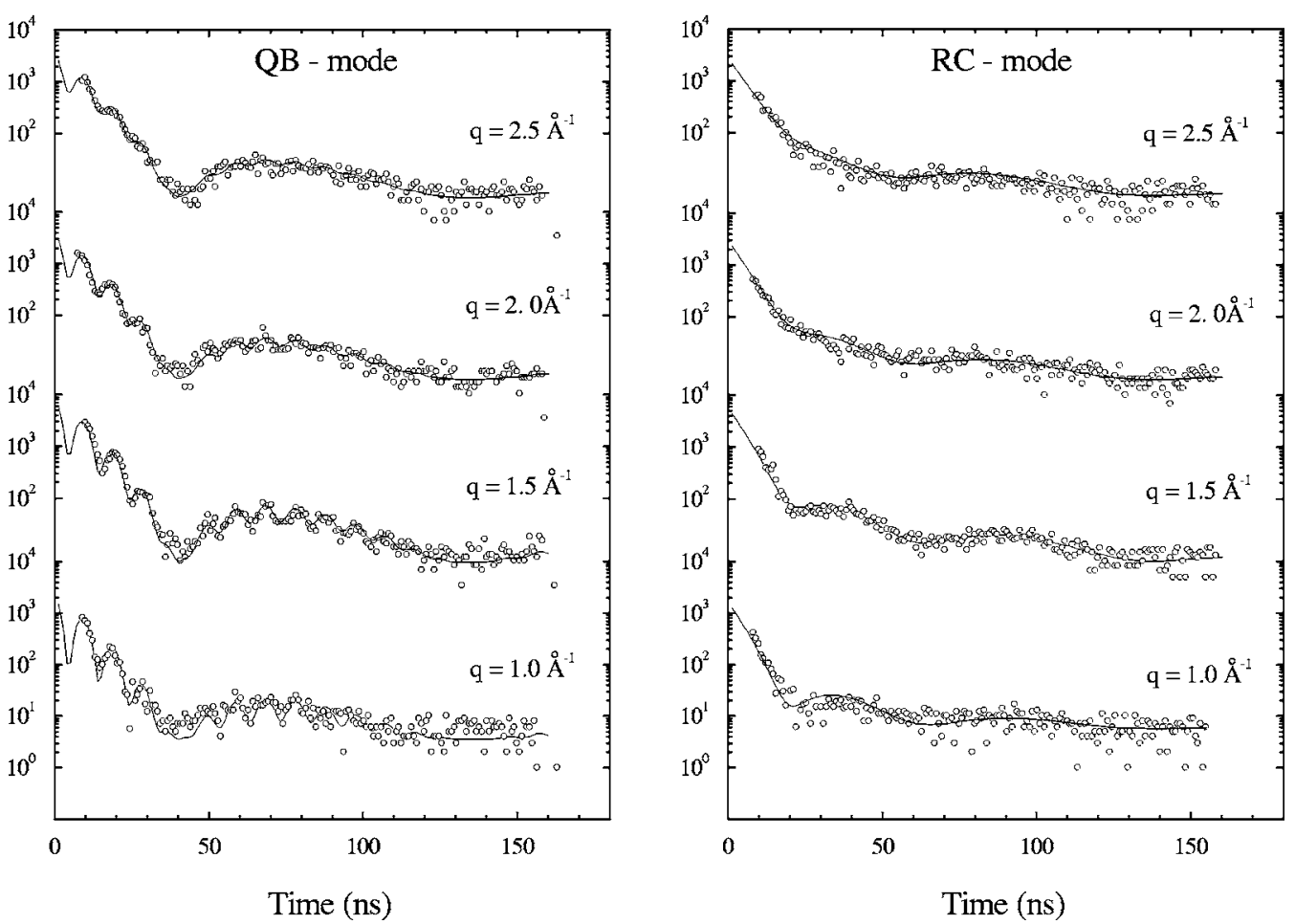

FIG. 4. Time dependences of scattering of the synchrotron radiation in the interferometer measured in the QB (left-hand panel) and RC (right-hand panel) regimes at the temperature of the glycerol sample, $255 \mathrm{~K}$, and at different momentum transfers. The solid curves are simulations obtained on the basis of Eqs. (8) and (9).

\section{MODEL EXPERIMENT FOR COMPARISON OF THE QB AND RC REGIMES OF NUCLEAR $\gamma$ RESONANCE TIME DOMAIN INTERFEROMETRY}

\section{A. Experimental setup}

To compare the feasibilities of the $\mathrm{QB}$ and $\mathrm{RC}$ regimes of TDI we chose glycerol as a sample for our experiments, a well-investigated glass-forming system. We studied the temperature and angular dependence of the quasielastic scattering from glycerol in parallel in the $\mathrm{QB}$ and $\mathrm{RC}$ regimes.

The experiments were performed at beamline ${ }^{21}$ ID-22N of the European Synchrotron Radiation Facility. The ring was operated in the 16-bunch mode, leaving windows of $\sim 176 \mathrm{~ns}$ for the observation of the delayed nuclear scattering. Preliminary tests of the equipment had been performed at the Petra1 beamline at HASYLAB (Hamburg). ${ }^{22}$ The experimental setup is sketched in Fig. 2.

The incident SR was monochromatized to $\sim 6 \mathrm{meV}$ bandwidth by a high-resolution monochromator using $\mathrm{Si}(422) \operatorname{Si}(1222)$ in nested geometry. Target $A$ was a stainless-steel (SS) foil of $\sim 6 \mu \mathrm{m}$ thickness, enriched to $95 \%$ in ${ }^{57} \mathrm{Fe}$. It was mounted on a Mössbauer driving unit, allowing one to move it at constant velocity of $\pm 9 \mathrm{~mm} / \mathrm{s}$ (corresponding to an energy shift of $\pm 90 \Gamma_{0}$ ) in the QB regime or to keep it at rest in the $\mathrm{RC}$ regime.

An identical SS foil was used as the second $\operatorname{target} B$ of the interferometer. Target $B$ was always kept at rest. It was inclined by $\sim 12^{\circ}$ in order to be perpendicular to the scattered radiation at the first structure factor maximum of glycerol.
The glycerol sample $S$ was mounted in between targets $A$ and $B$. It was of $\sim 4 \mathrm{~mm}$ thickness, kept in a sample holder with Kapton windows which was mounted at the cold finger of a liquid-nitrogen bath cryostat likewise equipped with Kapton windows.

The radiation scattered by $S$ into different scattering angles in the range $\sim 8^{\circ}-25^{\circ}$ was transmitted through target $B$. At a distance of $\sim 20 \mathrm{~cm}$ from the sample a multielement detector array (DA) with $4 \times 2$ avalanche photodiodes, each of area $1 \mathrm{~cm}^{2}$, was installed. ${ }^{23}$ The diodes at equal height and scattering angle were used in parallel. The four diode stripes intercepted radiation scattered from the sample into angles of $7.7^{\circ} \pm 1.3^{\circ}, 11.9^{\circ} \pm 1.3^{\circ}, 16.1^{\circ} \pm 1.3^{\circ}$, and $20.2^{\circ} \pm 1.3^{\circ}$, corresponding to $\mathbf{q}$ values of $0.977 \pm 0.17 \AA^{-1}, 1.517 \pm 0.17 \AA^{-1}$, $2.051 \pm 0.16 \AA^{-1}$, and $2.566 \pm 0.16 \AA^{-1}$, respectively.

The motion of $\operatorname{target} A$ was all times monitored by means of a similar SS target $C$ kept at rest in a far downstream position, where the interference of the nuclear forward scattering by targets $A$ and $C$ was recorded by an avalanche photodiode $D$.

Special care was taken to shield the detector array against radiation scattered by the surrounding. In particular a lead frame around $\operatorname{target} B$ was used as a shield against radiation scattered from the cryostat window and a baffle $b$ was mounted to shield the detector against scattering of the direct beam by air. In addition, $\operatorname{target} B$ was mounted in a way that it was not hit by the direct beam.

Also special care had been taken to minimize vibrations from the constant-velocity Mössbauer drive and from the 
$269 \mathrm{~K}$
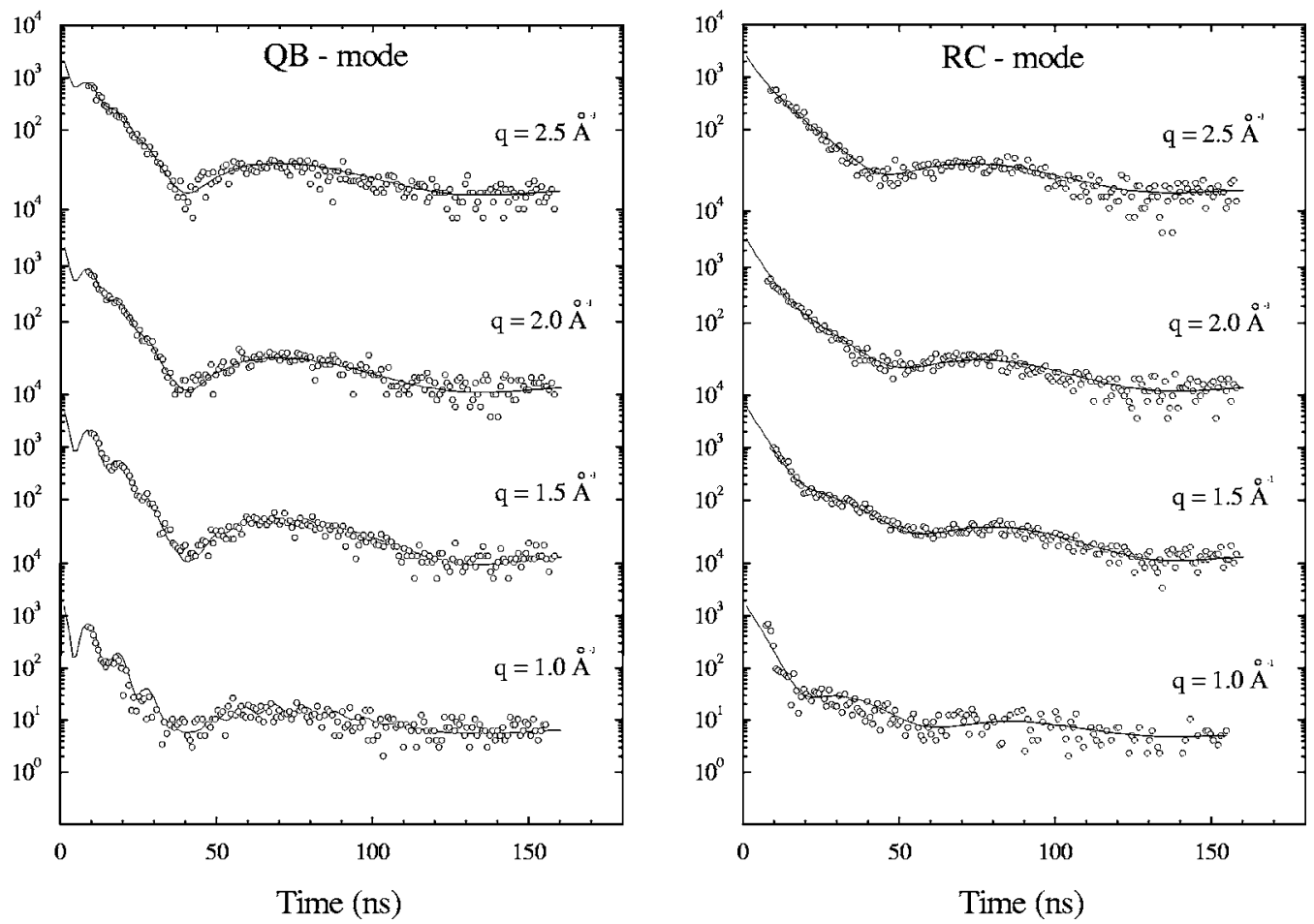

FIG. 5. Time dependences of scattering of the synchrotron radiation in the interferometer measured in the QB (left-hand panel) and RC (right-hand panel) regimes at the temperature of the glycerol sample, $269 \mathrm{~K}$, and at the different momentum transfers. The solid curves are simulations obtained on the basis of Eqs. (8) and (9).

cryostat vacuum pump, possibly affecting the triple system of the two targets and the scattering sample.

Delayed countrates were low and unstable, not exceeding $1 / \mathrm{s}$ in the structure factor maximum at $\sim 12^{\circ}$. The reason for both the low level and jumpy long-term instabilities could not be found during the experiment. Background with sample removed from the primary beam was $\sim 0.14$ /s.

\section{B. Experimental results and simulations}

The measurements were performed with the sample kept at temperatures $\theta \approx 140 \mathrm{~K}, 255 \mathrm{~K}$, and $269 \mathrm{~K}^{29}$ These temperatures lie around the mode-coupling glass transition temperature in glycerol $(\sim 228 \mathrm{~K})$, however below the melting point $\theta_{m}=291 \mathrm{~K}$. Thus the atomic dynamics at the start of structural relaxation in glycerol was investigated.

In spite of the uncomfortably low intensities, taking data for $\sim 9 \mathrm{~h}$ in each measurement session-i.e., for the particular interferometer regime (QB or $\mathrm{RC})$ and fixed temperature- the present experiment allowed one to demonstrate time-domain interferometry in the RC regime and to compare the RC and QB regimes. In Figs. 3-5 the interference patterns are shown which were obtained for the QB and $\mathrm{RC}$ regimes at different temperatures and for different momentum transfers.

In the observation time window the background consisted of a fast-decaying part reaching as far as $\sim 30 \mathrm{~ns}$ and a part which was constant over time. In Figs. 3-5 the fast-decaying part was substracted from the original data, whereas the con- stant background was left and was taken into account in the fitting.

At $140 \mathrm{~K}$ (see Fig. 3), where the scattering sample is stiff, the time evolutions in both regimes (left-hand and right-hand panels in the figures) correspond to the instrumental functions measured in the forward direction [compare Figs. 2(h) and 2(a) of Ref. 24]. It was possible to simulate the spectra at $140 \mathrm{~K}$ without assuming any additional line broadening for $\operatorname{target} A$. This proves the high quality of the constant-velocity drive and a sufficient shielding of the scattering system against vibrations from the drive and vacuum pump. In the QB regime the overall shape is determined by the dynamical beat (DB) characteristic of the propagation of radiation through the $\sim 6-\mu \mathrm{m}$-thick target (first minimum of the DB at $\sim 40 \mathrm{~ns}$ ). The overall dependence is strongly modulated by quantum beats. In the RC regime the dynamical beat pattern determining the overall shape of the time dependence is characteristic for a target of twice this thickness (first minimum of the DB at $\sim 20 \mathrm{~ns}$ ).

At higher temperatures (see Figs. 4 and 5), the sample exhibits dynamics, which modifies increasingly the interference patterns in both regimes. In the QB regime the beat pattern loses dramatically its contrast, whereas in the RC regime the overall shape of the interference pattern is strongly modified. The modifications of the interference pattern depend significantly on both sample temperature and scattering angle. Before proceeding to discuss these features we would like to make several comments concerning the slow atomic dynamics in our sample. 


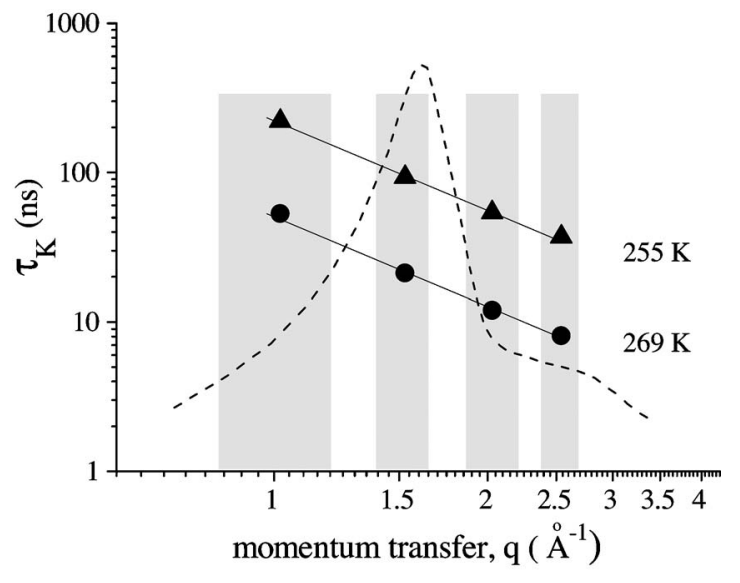

FIG. 6. Relaxation times obtained from the fit of the experimental time evolutions with the theory [Eqs. (8) and (9)] are plotted against momentum transfer $q$ for the two sample temperatures $255 \mathrm{~K}$ and $269 \mathrm{~K}$. For $140 \mathrm{~K}$, the relaxation times lie far beyond the scale of the figure. The solid lines indicate $\tau \sim q^{-2}$. The dashed line gives qualitatively the shape of the structure function for glycerol (Ref. 27). The shadowed bars indicated the $q$ ranges for the scattering accepted by the detector segments.

Glycerol near its transition to the glassy state exhibits structural relaxation on time scales comparable with the nuclear lifetime of ${ }^{57} \mathrm{Fe}$. This type of relaxation is related to the long-range transport of molecules in a medium. The structural relaxation in glycerol was studied by many methods (e.g., see Ref. 25 and references therein). Empirically it is described by a superposition of damped oscillators with a distribution of relaxation rates. The common description of this relaxation is based on the Kohlrausch stretched exponential with a particular stretching coefficient $\beta$, called the Kohlrausch exponent. The Kohlrausch exponent describes the deviation of the energy spectrum of a collection of damped oscillators from a Lorentzian shape. The intermediate scattering function in glycerol in the nanosecond to microsecond relaxation regime is thus represented by the following expression $^{25,1,18 \text { : }}$

$$
S(\mathbf{q}, t)=f_{D W} e^{-\left(t / \tau_{K}\right)^{\beta}} \quad \text { with } 0<\beta<1,
$$

where $f_{D W}$ is the Debye-Waller factor accounting for the thermal vibrations of the atoms and fast structural relaxations and $\tau_{K}$ is the relaxation time, which depends on momentum transfer and temperature. The Kohlrausch exponent can be a slightly varying function of temperature and momentum transfer. ${ }^{26}$ In our case of coherent scattering we employ the value of the stretching parameter found in Ref. 25 for coherent neutron scattering, $\beta=0.7$. That is, we assume that only the width, and not the shape, of the excitation spectrum is changing with temperature and momentum transfer. As concerns the Debye-Waller factor, we assumed that in our sample at the temperatures under study, it varies like in a harmonic solid. This means that in the expression $f_{D W}$ $=\exp \left\{-\left\langle r^{2}\right\rangle q^{2}\right\}$ the mean-square displacement was taken proportional to the temperature: $\left\langle r^{2}\right\rangle \propto \Theta$. We compare the values $f_{D W}=0.905$ for $q=1.43 \AA^{-1}$ in Ref. 25 and of $f_{D W}=0.87$ for
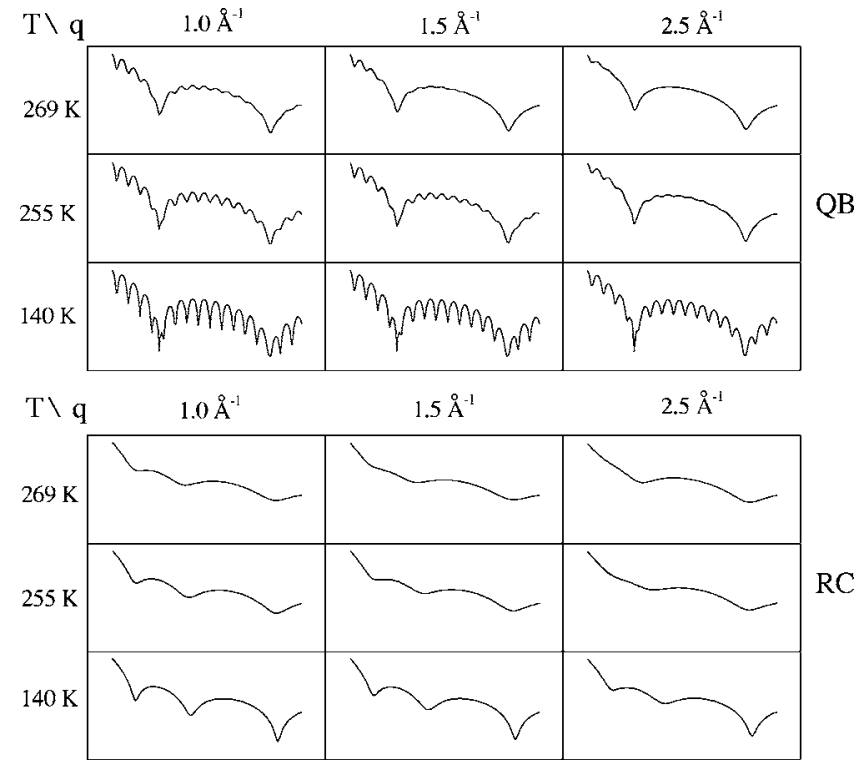

FIG. 7. Simulations of the interference patterns in the QB (the upper block of panels) and the RC (the lower block of panels) regimes at different temperatures of the glycerol sample and at different momentum transfers. The simulations fully correspond to the experimental dependences.

$q=1.53 \AA^{-1}$ in Ref. 1 for the temperature $\Theta=250 \mathrm{~K}$. When recalculated for the same momentum transfer $q=1.53 \AA^{-1}$ they differ by about $2 \%(0.89 / 0.87)$. This small difference could be just the isotope effect in predeuterated glycerol used in Ref. 25. We accepted the value $f_{D W}=0.87$ for this point. Then relying on the linear dependence of $\ln \left(f_{D W}\right)$ on temperature obtained in Ref. 25, Fig. 4(b), we calculated $f_{D W}$ for the other two temperatures and for the momentum transfers under study. The results are shown in Table I.

At the given limited statistics of the data, all time evolutions could be well simulated assuming the presented form of the intermediate scattering function and the accepted values for $\beta$ and $f_{D W}$. The only variable parameter was the relaxation time $\tau_{K}$. The important point is that it was possible to find sets of the relaxation times by which the two sets of completely different time evolutions for the QB and RC regimes were consistently fitted; see the solid curves in Figs. $3-5$. The relaxation times found from the fit are shown in Fig. 6. It is seen that the two sets of $\tau_{K}$ associated with temperatures $255 \mathrm{~K}$ and $269 \mathrm{~K}$ are arranged in a distinct order, where approximately the dependence $\tau_{K} \propto q^{-2}$ is fulfilled. Like in a previous study ${ }^{28}$ no increase of the relaxation time near the structural factor maximum at $\sim 12^{\circ}$ was observed.

TABLE I. Debye-Waller factors used for the simulation.

\begin{tabular}{lllll}
\hline \hline$\Theta(\mathrm{K}) / q\left(\AA^{-1}\right)$ & 0.977 & 1.517 & 2.051 & 2.566 \\
140 & 0.969 & 0.926 & 0.869 & 0.803 \\
255 & 0.944 & 0.870 & 0.775 & 0.671 \\
269 & 0.941 & 0.863 & 0.764 & 0.656 \\
\hline \hline
\end{tabular}



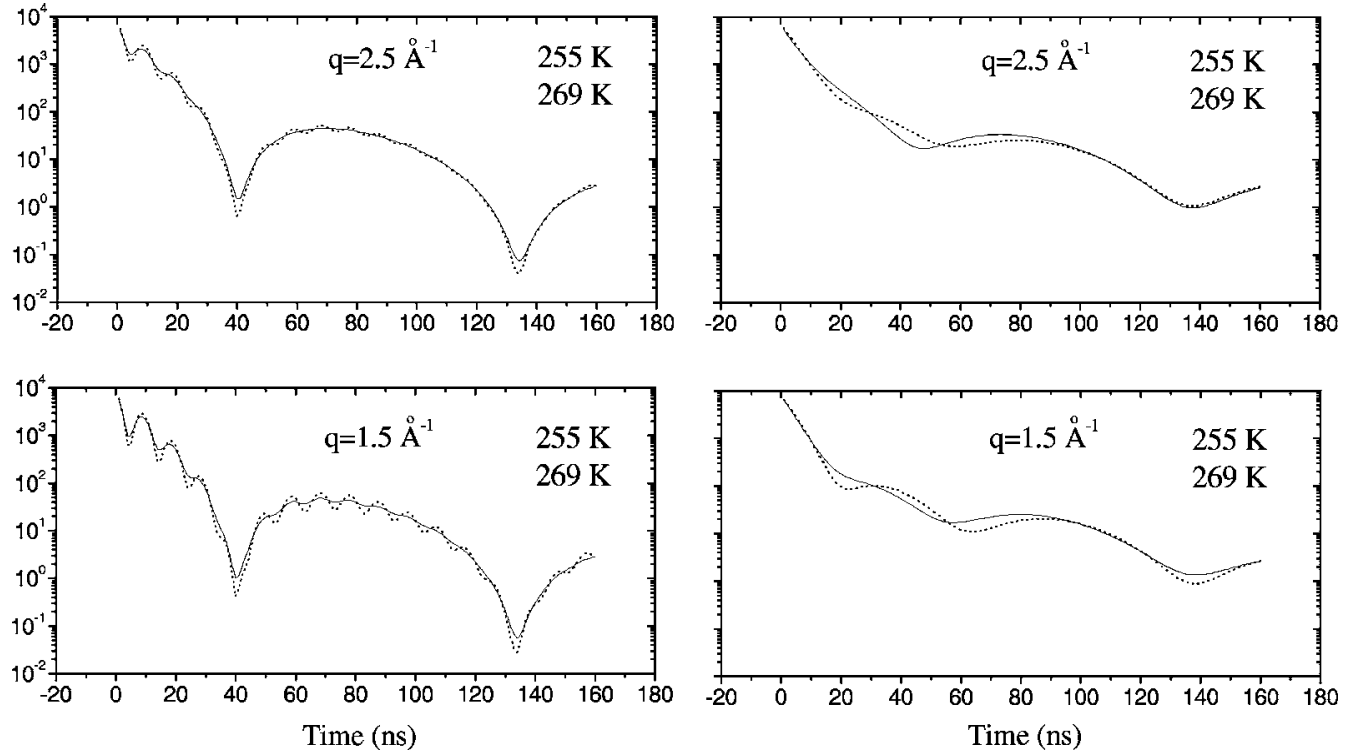

FIG. 8. Variations of the interference pattern are compared for the QB and RC regimes of the interferometer with a change of the temperature at the different momentum transfers.

\section{Analysis}

After normalization of the fit curves of our measurements to one and the same vertical scale we grouped them together into two $3 \times 3$ matrices, each referring to a particular regime of the interferometer. The rows of the matrices correspond to different temperatures, whereas the columns correspond to different momentum transfers. The result is represented in Fig. 7. The evolution of the interference patterns with temperature and momentum transfer is well seen in this figure.

In the QB regime the overall shape of the interference pattern remains unchanged for all scattering angles and temperatures; however, a strong modification of the QB pattern occurs (see the QB matrix). Two kinds of modifications can be distinguished: (1) a decrease of the initial QB contrast with the rise of temperature and momentum transfer and (2) a progressive fading of the $\mathrm{QB}$ contrast with time at higher temperatures and momentum transfers. The first effect is caused by the decrease of the Debye-Waller factor with the rise of temperature and momentum transfer; see Table I. With this the amplitude of the intermediate scattering function given by Eq. (10) is decreasing and, hence, the role of the modulated term in the summary picture described by Eq. (8) is diminished. The second effect is related to the decrease of the relaxation time with increasing temperature and momentum transfer. The decrease of the relaxation time causes an accelerated decay of the intermediate scattering function given by Eq. (10) and, hence, a progressive fading of the modulated term with time in Eq. (8). Similar transformations of the interference pattern were obtained in Ref. 1 in the QB regime with increasing temperature for fixed $q=1.5 \AA^{-1}$.

In the RC regime, by contrast, the overall shape of the interference pattern is modified. However, the changes of the intermediate scattering function are not that simply traced here as in the QB regime. The intermediate scattering function entering into each of the four terms of Eq. (9) modifies the interference pattern in a rather complicated way. The most pronounced effect of atomic dynamics is a dramatic reformation of the dynamical beat structure of the time dependence of the scattering intensity both with the rise of the temperature (from bottom to top in the RC matrix) and of the momentum transfer (from left to right in the RC matrix): the dynamical beat contrast is changed and the dynamical beats are shifted. In the extreme case, for $q \approx 2.5 \AA^{-1}$ and $\theta$ $=269 \mathrm{~K}$ the shape of the DB pattern approaches that characteristic of the single nuclear target (compare the shapes of the interference patterns in the top-right panels of the QB and RC matrices). A characteristic transformation from a doubletarget DB (the bottom-left panel in the RC matrix: first DB minimum at $\sim 20 \mathrm{~ns}$ ) to almost a single-target DB (the topright panel in the RC matrix: first DB minimum at $\sim 40 \mathrm{~ns}$ ) due to atomic dynamics in the sample was observed. The essential reason for such modifications is the perturbation of the radiative coupling of the nuclear targets and hence the variation of the conditions of nuclear multiple scattering in the system.

Thus in the two regimes of time-domain interferometry the increase of quasielastic scattering is revealed in quite different ways: either through pronounced changes of the quantum beat modulation of a fixed interference pattern (in the QB regime) or via strong changes of the interference pattern itself, mainly of its dynamical beat structure (in the $\mathrm{RC}$ regime).

As for the sensitivity of time-domain interferometry in the two different regimes, the following holds. Since the QB is a very pronounced feature of the time evolution measured in the QB regime, its fading due to sample dynamics is more easily recognized during the experiment than the changes of the interference pattern in the $\mathrm{RC}$ regime. The analysis according to the theory, by contrast, is more sensitive to relaxation parameters when the time evolutions were measured in the RC regime. In Fig. 8 the variations of the interference patterns are compared for the same variations of temperature for two different values of $q$. The comparison shows that the $\mathrm{RC}$ regime has especially high sensitivity to strong dynam- 
ics, which in the QB regime leads to a very fast fading of the QB contrast (practically within the time range unaccessible in the experiment), but is seen in the $\mathrm{RC}$ regime in the full time window. Therefore one can conclude that the consistent fit of the data sets obtained in the measurements in the QB and $\mathrm{RC}$ regimes can essentially increase the reliability of the data evaluation and extend the observed range of dynamics towards higher atomic mobility.

\section{ACKNOWLEDGMENTS}

This work was supported by the Bundesministerium für Bildung, Wissenschaft, Forschung and Technologie, under Contract No. $05 \mathrm{KS} 1 \mathrm{WOC} / 2$, and by the RRC Kurchatov Institute. G.V.S. gratefully acknowledges the financing of a stay at the TUM by the Deutsche Forschungsgemeinschaft.
*Electronic address: smirnov@polyn.kiae.su

${ }^{1}$ A. Q. R. Baron, H. Franz, A. Meyer, R. Rüffer, A. I. Chumakov, E. Burkel, and W. Petry, Phys. Rev. Lett. 79, 2823 (1997).

${ }^{2}$ G. V. Smirnov, Hyperfine Interact. 123/124, 31 (1999).

${ }^{3}$ G. T. Trammell and J. P. Hannon, Phys. Rev. B 18, 165 (1978).

${ }^{4}$ Y. Kagan, A. M. Afanas'ev, and V. G. Kohn, Phys. Lett. 68A, 339 (1978).

${ }^{5}$ Y. Kagan, A. M. Afanas'ev, and V. G. Kohn, J. Phys. C 12, 615 (1979).

${ }^{6}$ S. Kikuta, Y. Yoda, Y. Hasegawa, K. Izumi, T. Ishikawa, X. W. Zhang, S. Kishimoto, H. Sugiyama, T. Matsushita, M. Ando, C. K. Suzuki, M. Seto, H. Ohno, and H. Takei, Hyperfine Interact. 71, 1491 (1992).

${ }^{7}$ M. Pleines, R. Lübbers, M. Strecker, G. Wortmann, O. Leupold, Y. V. Shvyd'ko, E. Gerdau, and J. Metge, Hyperfine Interact. 120/121, 181 (1999).

${ }^{8}$ G. V. Smirnov, U. van Bürck, J. Arthur, S. L. Popov, A. Q. R. Baron, A. I. Chumakov, S. L. Ruby, W. Potzel, and G. S. Brown, Phys. Rev. Lett. 77, 183 (1996).

${ }^{9}$ P. Schindelmann, U. van Bürck, W. Potzel, G. V. Smirnov, S. L. Popov, E. Gerdau, Yu. V. Shvyd'ko, J. Jäschke, H. D. Rüter, A. I. Chumakov, and R. Rüffer, Phys. Rev. A 65, 023804 (2002).

${ }^{10}$ W. Potzel, U. van Bürck, P. Schindelmann, H. Hagn, G. V. Smirnov, S. L. Popov, E. Gerdau, Yu. V. Shvyd'ko, J. Jäschke, H. D. Rüter, A. I. Chumakov, and R. Rüffer, Hyperfine Interact. 151/152, 263 (2003).

${ }^{11}$ G. V. Smirnov, U. van Bürck, W. Potzel, P. Schindelmann, S. L. Popov, E. Gerdau, Y. V. Shvyd'ko, H. D. Rüter, and O. Leupold, Phys. Rev. A 71, 023804 (2005).

${ }^{12}$ R. Callens, R. Coussement, T. Kawakami, J. Ladrière, S. Nasu, T. Ono, I. Serdons, K. Vyvey, T. Yamada, Y. Yoda, and J. Odeurs, Phys. Rev. B 67, 104423 (2003).

${ }^{13}$ W. Sturhahn, C. L'abbé, and T. S. Toellner, Europhys. Lett. 66, 506 (2004).

${ }^{14}$ R. Callens, C. L'abbé, J. Meersschaut, I. Serdons, W. Sturhahn, and T. S. Toellner, Phys. Rev. B 72, 081402(R) (2005).

${ }^{15}$ G. V. Smirnov, V. G. Kohn, and W. Petry, Phys. Rev. B 63,
144303 (2001).

${ }^{16}$ H. Jex, A. Ludwig, F. J. Hartmann, E. Gerdau, and O. Leupold, Europhys. Lett. 40, 317 (1997).

${ }^{17}$ A. Ludwig and H. Jex, Physica B 254, 1 (1998).

${ }^{18}$ H. Franz, W. Petry, and A. Q. R. Baron, Hyperfine Interact. 123/ 124, 865 (1999).

${ }^{19}$ M. Kaisermayr, B. Sepiol, H. Thiess, G. Vogl, E. E. Alp, and W. Sturhahn, Eur. Phys. J. B 20, 335 (2001).

${ }^{20}$ L. van Hove, Phys. Rev. 95, 249 (1954).

${ }^{21}$ R. Rüffer and A. I. Chumakov, Hyperfine Interact. 97/98, 589 (1996).

${ }^{22}$ H. Franz, B. Hukelmann, and J. R. Schneider, Hyperfine Interact. 126, 397 (2000).

${ }^{23}$ H. Franz, M. von Zimmermann, A. Ehnes, R. Nowak, J. Heuer, and E. Weckert (unpublished).

${ }^{24}$ U. van Bürck, W. Potzel, P. Schindelmann, Y. V. Shvyd'ko, E. Gerdau, O. Leupold, and H. D. Rüter, Phys. Rev. A 61, 013803 (1999).

${ }^{25}$ J. Wuttke, W. Petry, and S. Pouget, J. Chem. Phys. 105, 5177 (1996).

${ }^{26}$ J. Wuttke, W. Petry, G. Coddens, and F. Fujara, Phys. Rev. E 52, 4026 (1995).

${ }^{27}$ M. Soltwisch, M. Elwenspoek, and D. Quitmann, Mol. Phys. 34, 33 (1977).

${ }^{28}$ H. Franz, A. Baron, R. Rüffer, W. Petry, and E. Burkel (unpublished).

${ }^{29}$ The temperatures at the sensor position in the experiment were $128,230,240 \mathrm{~K}$. These were obviously shifted from the real temperatures at the sample, possibly due to a high leakage of the bath cryostat. To obtain consistency with relaxation times obtained for the same sample in previous work (Ref. 1), we have recalibrated our temperature scale and found temperatures of $140,255,269 \mathrm{~K}$. The uncertainty in determining the absolute temperature has no influence on the $\mathbf{q}$ dependence or on the comparison of the RC and $\mathrm{QB}$ regimes performed at equal temperatures. 